\title{
Concepts and Applications of Ultrafast Cardiac Ultrasound Imaging
}

\author{
Aniela Petrescu ${ }^{133)}$, Jan D’hooge ${ }^{1)}$, Jens-Uwe Voigt ${ }^{12)}$ \\ 1) University of Leuven, Belgium \\ 2) University Hospital Leuven, Belgium \\ 3) University Hospital Mainz, Germany
}

Address for correspondence

Prof. Dr. med. Jens-Uwe Voigt

Department of Cardiovascular Diseases, University Hospital Leuven

Department of Cardiovascular Sciences, University Leuven

Herestraat 49, 3000 Leuven, Belgium

iens-uwe.voigt@uzleuven.be

Tel.: +32 16349016

Key Words

new technology, high frame rate imaging, shear wave imaging 


\begin{abstract}
The concept of ultrafast echocardiographic imaging has been around for decades. However, only recent progress in ultrasound machine hardware and computer technology allowed to apply this concept to echocardiography. High frame rate echocardiography can visualize phenomena that have never been captured before. It enables a wide variety of potential new applications, including shear wave imaging, speckle tracking, ultrafast Doppler imaging and myocardial perfusion imaging. The principles of these applications and their potential clinical use will be presented in this manuscript.
\end{abstract}




\section{Introduction}

Despite the rapid growth of multimodality imaging, echocardiography is still the main tool for non-invasive assessment of the cardiovascular system, given its high clinical value, low cost and wide availability. Also in terms of temporal resolution, echocardiography stands up among other cardiac imaging modalities ${ }^{1}$. Current commercial echocardiographic imaging systems operate on a basis of approximately 60 frames per second for $2 \mathrm{D}$ imaging which allows a good visualisation of heart motion and morphology, as well as the assessment of certain functional aspects during the cardiac cycle. However, in order to understand shortlived cardiac motion events, a higher temporal resolution is required ${ }^{2,3}$. Recent advances in ultrasound technology and computational power have facilitated the implementation of ultrafast cardiac ultrasound imaging, which allows imaging of the heart at up to 5000 frames per second, close to hundred times more than with conventional echocardiography. The acquisition of such high frame rate data can complement current echocardiographic techniques by providing new insights into myocardial properties and function as well as cardiac blood flow patterns.

Currently, ultrafast ultrasound imaging is at a clinical proof-of-concept stage but shows amazing and promising first results. In the current review we provide a brief overview of the principles and concepts of cardiac ultrafast imaging and discuss some of the potential clinical applications of this technique.

\section{High Frame Rate Imaging}

Ultrasound imaging is based on sound waves which are emitted into the tissue and then return to the transducer as reflected echoes. In conventional imaging, focussed ultrasound beams are sequentially sent in different directions and their echoes are used to reconstruct a respective line of the image sector. Given a velocity of sound in soft tissue of about $1,530 \mathrm{~m} / \mathrm{s}$, and an image depth of $15 \mathrm{~cm}$ (round-trip distance $30 \mathrm{~cm}$ ), one single pulseecho takes $\approx 200 \mu$ s before it returns to the transducer. An image composed of 180 lines would then require $36 \mathrm{~ms}$ allowing a frame rate of ca. 28 frames per second. Frame rate can only be increased if the density of scan lines or the image depth are reduced ${ }^{4}$. This trade-off between frame rate, depth and number of lines is a frequent challenge in the use of echocardiographic imaging, since both, good temporal and spatial resolution are desired.

The concept of ultrafast echocardiographic imaging was suggested more than thirty years $\mathrm{ago}^{5}$. A commonly-used technique is multiline acquisition (MLA), which is implemented on most currently-available systems. In this approach, sent beams are less focussed which allows reconstructing two or more scan lines simultaneously through differential focussing on 
receive. In this way, the currently typical 60 frames per second of modern ultrasound systems are reached ${ }^{6}$.

The concept can be extrapolated to sending one completely unfocussed ultrasound pulse in all directions, while focussing on scan lines happens only during receive. This socalled Diverging Wave Imaging (DWI) allows to use every single pulse to produce one image, thus reaching frame rates in the range of 5000 frames per second ${ }^{7,8}$. This, however, comes at the cost of a low signal-to-noise ratio, and consequently lower images quality, as the transmitted sound energy per scan line is much lower. In order to raise image quality again, coherent compounding of several successive diverging waves is used ${ }^{9,10,11}$.

While many aspects of myocardial mechanics can be analysed with conventional echocardiographic imaging, higher temporal resolution offers additional information. We will show in the following how increasing the frame rate paves the way to fascinating new applications of echocardiography such as myocardial stiffness measurements, imaging of coronary flow or visualizing intracavitary blood flow.

\section{Assessment of fast tissue motion}

\section{Shear Wave Imaging}

Shear Wave Imaging (SWI) utilizes ultrafast ultrasound imaging to image the propagation of shear waves which can occur either naturally (e.g after closure of cardiac valves) or can be artificially induced (by direct mechanical excitation or by using the acoustic radiation force of strong ultrasound pulses) ${ }^{12-15}$ Figure [F1]). Shear waves then propagate with a velocity that is directly related to tissue stiffness Figure [F2]). In a linear, isotropic, elastic medium, the velocity of shear waves $(\boldsymbol{c})$ can be expressed as:

$$
c=\sqrt{\frac{\mu}{\rho}}
$$

where $\boldsymbol{\mu}$ is the shear modulus (i.e., stiffness) and $\rho$ is the density of the tissue ${ }^{16}$. Shear wave velocities in the myocardium are typically in the range of 1 to $5 \mathrm{~m} / \mathrm{s}^{13}$.

The clinical potential of this approach has been demonstrated in various fields of medicine including breast lesions detection ${ }^{17}$, liver fibrosis staging ${ }^{18}$ and the assessment of arterial stiffness $^{19}$. Due to technical challenges related to image depth, intercostal sector imaging and a continuously moving target, first implementations of cardiac SWI have occurred only recently and studies investigating the use of SWI to quantify myocardial stiffness were initially mainly performed in open-chest animal models ${ }^{20-22}$. Improvements in ultrasonography techniques demonstrated lately also the feasibility of this method in in-vivo closed-chest animals and humans $\mathbf{s}^{12,15,23,24}$. 


\section{Stiffness measurements to assess systolic and diastolic function}

Assessment of left ventricular function is fundamental in the understanding of cardiovascular pathophysiology. Conventional echocardiographic parameters allow quantification of systolic and diastolic function based on the visualisation and interpretation of changes in volumes and velocity measurements of the blood ${ }^{25,26}$. However, none of these parameters can measure cardiac relaxation and contractility directly. Higher frame rates offer the possibility to visualize fast myocardial tissue movements that cannot be detected by conventional echocardiography. Therefore, by studying the speed of propagation of shear waves in the myocardium, the intrinsic property of tissues that reflect their composition and elasticity (i.e myocardial stiffness) can be measured ${ }^{23,27}$. During early diastole, myocardial stiffness reflects the pure passive elastic properties of the myocardium. During systole, contractile forces add up, thereby reflecting the intrinsic contractile state of the myocardium ${ }^{28}$. Therefore, local measurements of myocardial stiffness during systole or diastole would allow an assessment of systolic and diastolic properties of the heart. Shear waves can be artificially generated throughout the cardiac cycle, allowing the quantification of myocardial stiffness variations in all systole and diastole. On the other side, the assessment of natural shear wave velocities is limited to the time point of the physiologic event, mostly the closure of the aortic or mitral valve.

\section{Artificial shear waves}

By using ultrasonic push pulses, Pernot et al. showed, that, similar to end-systolic elastance, myocardial stiffness measured during systole reflects the intrinsic contractile state of the myocardium in Langendorff perfused isolated rat hearts ${ }^{29}$. The stiffness was determined by inducing a shear wave with a $300 \mu$ s excitation "push" pulse and consecutive diverging wave imaging with a cardiac phased array transducer $(2.75-\mathrm{MHz})$ connected to a SuperSonic Imagine Scanner (Aix-en-Provence, France). The same group also evaluated the potential of shear wave imaging to quantify passive diastolic myocardial stiffness in an ovine model of ischemic cardiomyopathy, and they could show that SWI can differentiate between stiff infarcted wall and softer wall containing stunned myocardium ${ }^{30}$. Moreover, they have used the differing stiffness of the myocardium in different directions and layers to assess myocardial fiber architecture ${ }^{31}$.

More recently, transthoracic SWI with extrinsic push pulses was shown to be feasible in humans ${ }^{15,32}$. Villemain et al. investigated the diastolic myocardial stiffness in healthy volunteers of three age groups ( 20 to 39 years, $n=20 ; 40$ to 59 years, $n=20$; and 60 to 80 years, $n=20)$ and in hypertrophic cardiomyopathy $(\mathrm{HCM})$ patients with heart failure and preserved ejection fraction (HFpEF). Myocardial stiffness increased significantly with age 
$(2.59 \pm 0.58 \mathrm{kPa}, 4.70 \pm 0.88 \mathrm{kPa}$, and $6.08 \pm 1.06 \mathrm{kPa}$, respectively; $\mathrm{p}<0.01$ between each group) and showed a significant correlation with classic echocardiographic parameters of diastolic function (E/A ratio, $r=0.55$; left atrial volume index, $r=0.67 ; E / e^{\prime}$ ratio, $r=0.62 ; E / V p$ ratio, $r=0.50)$. Furthermore, myocardial stiffness was significantly higher in HCM patients than in healthy volunteers $(\mathrm{HV})(12.68 \pm 2.91 \mathrm{kPa} \text { vs. } 4.47 \pm 1.68 \mathrm{kPa} \text {, respectively; } \mathrm{p}<0.01)^{16}$. The authors reported the optimal cut-off value of myocardial stiffness for detection of HCMHFpEF to be $8 \mathrm{kPa}$ (Figure [F3]).

SWI by using ultrasonic push pulses has the advantage of being able to estimate the stiffness of soft tissue at any time during the cardiac cycle. However, push pulses require a high power output from the transducer. Given the small foot print of transthoracic transducers, the power density is very high, warming becomes an issue, and the probes operate at the limits of what is technically feasible. In general, only shear wave generation close to the transducer appears feasible with current technology. Further, the excited shear waves are small in amplitude $\left(\sim 1 \mu \mathrm{m}^{33}\right)$ and attenuate fast when propagating through the tissue which makes the estimation of their propagation speed challenging.

\section{Natural shear waves}

Natural shear waves are produced by physiologic events- They are characterized by higher displacements $\left(\sim 100 \mu \mathrm{m}^{12}\right.$ versus $\left.\sim 1 \mu \mathrm{m}^{33}\right)$ which allows their detection over larger distances and in noisier conditions, making this approach more suitable for closed chest myocardial stiffness estimation. Kanai first investigated shear waves induced by the closure of the aortic valve and measured their propagation velocities in the interventricular septum ${ }^{34}$. The hypothesis that there is a correlation between the velocity of the shear waves and the myocardial stiffness that could have important diagnostic and therapeutic implications led to a significant progress over the past few years. Several studies have shown the propagation speed of natural shear waves to be in the range of 3-4 m/s after aortic valve closure and 2 to $3 \mathrm{~m} / \mathrm{s}$ after mitral valve closure in young healthy volunteers. It becomes significantly higher with ageing, as well as in pathology such as cardiac amyloidosis, hypertrophic cardiomyopathy and arterial hypertension ${ }^{16,23,35}$. The propagation speed of shear waves after mitral valve closure (i.e end-diastole) appeared to be related to the degree of diastolic dysfunction and showed a good correlation with E/E', as well defined standard echocardiographic parameter for predicting diastolic filling pressures ${ }^{23}$. We further showed that in heart transplant recipients, end-diastolic shear wave propagation velocities have a good correlation with parameters of cardiac magnetic resonance used as marker of diffuse myocardial injury Figure [4], and were the best non-invasive parameter to correlate with pulmonary wedge pressures measurements, as a reflection of an increase in left ventricular filling pressures ${ }^{24}$. 
Assessing shear waves with 2D imaging detects only the projection of the shear wave velocity vector on the image plane. High frame rate 3D mapping techniques allow to display the true propagation direction. This may allow to investigate the origin of natural shear waves and the characterization of left ventricular myocardial stiffness in different directions ${ }^{36-40}$. An example of a mechanical wave in the heart visualized with fast 3D imaging technology is given in Figure[F5] $]^{36}$.

The quantitative estimation of myocardial stiffness by non-invasive SWI without the need for CMR or histology could be clinically useful for characterizing diffuse myocardial fibrosis, ischemic scar and other pathology. It could also be utilized for a non-invasive assessment of myocardial systolic and diastolic function. Further clinical validation is needed, however, before this promising technique can be applied in the clinical routine.

\section{Ultrafast Speckle-Tracking Echocardiography}

Speckle tracking echocardiography (STE) can offer valuable diagnostic and prognostic information and is increasingly performed in many echocardiography laboratories worldwide ${ }^{41}$. The global and segmental measurement of myocardial strain allows a better understanding of cardiac mechanics than conventional echocardiographic parameters, however, it relies on the frame rate of the underlying grey scale image, typically around $60 \mathrm{~Hz}$. While this is sufficient for the assessment of myocardial motion and deformation (strain), an accurate measurement of velocity and strain rate during fast and short-lived events of the cardiac cycle is impossible. Tissue Doppler ultrasound with frame rates up to 300 frames per second would be a valid alternative, but it is limited by its one-dimensional assessment of tissue velocities only along the ultrasound beam. Hence, dedicated HFR STE algorithms are being developed in order combine the high temporal resolution of ultrafast ultrasound imaging with the $2 \mathrm{D}$ information of speckle tracking algorithms ${ }^{42-45}$. Thus, high frame rate imaging might lead to the incorporation of STE in the routine stress- of fetal echocardiography, that certainly benefit from temporally-resolved myocardial deformation ${ }^{4,42,43}$.

\section{Advanced Cardiac Flow Imaging}

\section{Coronary Ultrafast Doppler Angiography (CUDA)}

The evaluation of coronary flow reserve during a coronary angiography is an important tool in the diagnostic of ischemic heart disease, however, it is invasive and not without risks. Imaging of coronary arteries with computed coronary angiography ${ }^{46}$ offers new possibilities 
for the anatomical and functional assessment of coronary arteries, but it is also ionizing and requires contrast agents.

HFR Imaging has also been tested to characterize myocardial perfusion using Doppler techniques. By using specific spatiotemporal clutter filters 47, Ultrasound Doppler Angiography (CUDA) can distinguish the myocardium with high echogenicity and relatively slow motion from blood vessels with low echogenicity and faster motion ${ }^{48}$. This provides the detection of coronary flow in epicardial and intramural arteries with characterization of coronary flow reserve and intramural perfusion. Maresca et al. performed the first experiments in the beating heart of large animal models showing that CUDA accurately quantifies the change of flow rate in coronary arterioles (with diameters ranging from 500 to $100 \mu \mathrm{m}$ ) during hyperemia when compared to gold standard measurements provided by a proximal flow meter probe placed on the epicardial artery (Figure [F6]) ${ }^{47}$. However, imaging a 3D vascular network by a 2D Doppler approach does not allow a reliable quantification of the coronary flow rate due to angular dependence of axial Doppler estimates. In order to overcome this limitation, 3D ultrafast CUDA was recently introduced to image the coronary vasculature in three-dimensions and quantify the absolute coronary volumetric flow rates non-invasively ${ }^{49}$.

CUDA is a contrast agent-free, non-invasive method to map the coronary vasculature allowing the non-invasive assessment of intramural perfusion and coronary flow variation. These results could open new possibilities for the characterization of intramural perfusion in patients with microvascular angina.

\section{Ultrafast Vector Flow Imaging}

Conventional echocardiographic colour Doppler measures flow velocities only along the direction of the ultrasound beam. It is further limited by aliasing effects and low Doppler frame rates ${ }^{50}$. Echocardiographic particle image velocimetry (echo-PIV) is capable of assessing blood motion in any direction within the B-mode image plane based on the tracking of ultrasound contrast agent echoes from frame to frame and obtain displacement vectors $^{51,52}$. Clinical limitations of echo-PIV are the need for the intravenous administration of gas-filled microbubbles, which is expensive and time consuming, as well as the far too low frame rate of regular grey scale imaging, which limits velocity assessment to under $40 \mathrm{~cm} / \mathrm{s}^{53}$. Vector flow mapping (VFM) had been introduced as an alternative method of processing Doppler information in a way that velocity vectors of any direction within the image plane can be displayed ${ }^{54}$. However, the method relies on a number of assumption about the flow field which might not hold true in all circumstances and suffers from the low time resolution of the flow data $\operatorname{set}^{55}$. 
High frame rate imaging offers new possibilities for $2 \mathrm{D}$ and $3 \mathrm{D}$ blood flow estimation ${ }^{56}$. The high temporal resolution enables the measurement of a much wider range of velocities and, by using the speckle tracking approach, it allows to generate flow maps for all dimensions of the image data set without mathematical assumptions. Contrast agents are not necessary since reflections from blood cells are tracked. High frame rate imaging based Blood Speckle Tracking (BST) is therefore a novel blood flow visualization that might be used to characterize the hemodynamic consequences of valvulopathies and complex shunt lesions such as atrial or ventricular septal defects ${ }^{57,58}$. By visualizing 2D or 3D intracardiac flows, BST can be used to map, quantify and analyze complex flow patterns, e.g. in pediatric cardiology ${ }^{59-61}$. Different parameters that describe vortex formations of the blood inside the left ventricle have been proposed for the evaluation of blood flow energetics, including vortex position and area, formation time, duration during the cardiac cycle and relative strength ${ }^{59}$. Changes in intraventricular flow can be a marker of reduced ventricular function. Vortex visualization in fetal cardiac ventricles could contribute to a better evaluation of cardiac function before birth and might improve prenatal detection of coarctation of aorta. The feasibility of 3D blood speckle tracking for cardiac 4D acquisition was also investigated in the adult heart (Figure [F7] $)^{60-62}$. Although the feasibility of this method has been proven in several pediatric studies, the limited penetration depth and signal to noise ratio of high frame rate images limits the current application of blood speckle tracking techniques in adults.

\section{Summary}

The concept of ultrafast ultrasound has facilitated new applications in medical imaging. The high temporal resolution allows new insights into myocardial mechanics and hemodynamics. At the moment, the new technique is in an early phase and still suffers from some childhood diseases, such as limited penetration depth and image quality. However, for some clinical applications, the acquisition of temporally-resolved data may already now outweigh these limitations. Furthermore, technological developments will help to improve image quality in the future. Further studies are needed to explore the best clinical application of these high frame rate based techniques. Current preliminary results are already promising and suggest that high frame rate imaging will complement our echocardiographic toolbox in the coming years. 


\section{References}

1. BN S. Echocardiography in the era of multimodality cardiovascular imaging. Biomed Res Int. 2013;2013:1-11. doi:10.1155/2013/310483

2. Bercoff J, Tanter M, Fink M. Supersonic shear imaging: A new technique for soft tissue elasticity mapping. IEEE Trans Ultrason Ferroelectr Freq Control. 2004;51(4):396-409. doi:10.1109/TUFFC.2004.1295425

3. Bercoff J, Montaldo G, Loupas T, et al. Ultrafast compound doppler imaging: Providing full blood flow characterization. IEEE Trans Ultrason Ferroelectr Freq Control. 2011;58(1):134-147. doi:10.1109/TUFFC.2011.1780

4. Cikes M, Tong L, Sutherland GR, D'Hooge J. Ultrafast cardiac ultrasound imaging: Technical principles, applications, and clinical benefits. JACC Cardiovasc Imaging. 2014;7(8):812-823. doi:10.1016/j.jcmg.2014.06.004

5. Bruneel C, Torguet R, Rouvaen KM, Bridoux E, Nongaillard B. Ultrafast echotomographic system using optical processing of ultrasonic signals. Appl Phys Lett. 1977;30(8):371-373. doi:10.1063/1.89436

6. Tong L, Hamilton J, Jasaityte R, Cikes M, Sutherland GR, D'hooge J. Plane wave imaging for cardiac motion estimation at high temporal resolution: A feasibility study invivo. Ultrason Symp (IUS), 2012 IEEE Int. 2012:228-231.

doi:10.1109/ULTSYM.2012.0057

7. Brekke B, Nilsen LCL, Lund J, et al. Ultra-high frame rate tissue Doppler imaging. Ultrasound Med Biol. 2014;40(1):222-231. doi:10.1016/j.ultrasmedbio.2013.09.012

8. Kanai H. Propagation of Spontaneously Actuated Pulsive Vibration in Human Heart Wall and In Vivo Viscoelasticity Estimation. ieee Trans Ultrason Ferroelectr Freq Control. 2005;52(11). http://www.ecei.tohoku.ac.jp/hkanai/pdffullpaper/105kanai2005.pdf. Accessed March 19, 2018.

9. Tanter M, Bercoff J, Sandrin L, Fink M. Ultrafast compound imaging for 2-D motion vector estimation: application to transient elastography. IEEE Trans Ultrason Ferroelectr Freq Control. 2002;49(10):1363-1374.

http://www.ncbi.nlm.nih.gov/pubmed/12403138.

10. Caenen A, Pernot M, Kinn Ekroll I, et al. Effect of Ultrafast Imaging on Shear Wave Visualization and Characterization: An Experimental and Computational Study in a Pediatric Ventricular Model. App/ Sci. 2017;7(8):840. doi:10.3390/app7080840ї 
11. Papadacci C, Pernot M, Couade M, Fink M, Tanter M. High-contrast ultrafast imaging of the heart. IEEE Trans Ultrason Ferroelectr Freq Control. 2014;61(2):288-301. doi:10.1109/TUFFC.2014.6722614

12. Vos HJ, van Dalen BM, Heinonen I, et al. Cardiac shear wave velocity detection in the porcine heart. Ultrasound Med Biol. 2017;43(4):753-764.

doi:10.1016/j.ultrasmedbio.2016.11.015

13. Santos P, Petrescu A, Pedrosa J, et al. Natural shear wave imaging in the human heart: normal values, feasibility and reproducibility. IEEE Trans Ultrason Ferroelectr Freq Control. 2018:1-1. doi:10.1109/TUFFC.2018.2881493

14. Couade M, Pernot M, Messas E, et al. In Vivo Quantitative Mapping of Myocardial Stiffening and Transmural Anisotropy During the Cardiac Cycle. IEEE Trans Med Imaging. 2011;30(2):295-305. doi:10.1109/TMI.2010.2076829

15. Villemain O, Correia M, Khraiche D, et al. Myocardial Stiffness Assessment Using Shear Wave Imaging in Pediatric Hypertrophic Cardiomyopathy. JACC CardiovasC Imaging. November 2017. doi:10.1016/j.jcmg.2017.08.018

16. Villemain O, Correia M, Mousseaux E, et al. Myocardial Stiffness Evaluation Using Noninvasive Shear Wave Imaging in Healthy and Hypertrophic Cardiomyopathic Adults. JACC Cardiovasc Imaging. March 2018. doi:10.1016/j.jcmg.2018.02.002

17. Gheonea IA, Stoica Z, Bondari S. Differential diagnosis of breast lesions using ultrasound elastography. Indian J Radiol Imaging. 2011;21(4):301. doi:10.4103/09713026.90697

18. Lee SM, Lee JM, Kang HJ, et al. Liver fibrosis staging with a new 2D-shear wave elastography using comb-push technique: Applicability, reproducibility, and diagnostic performance. PLoS One. 2017;12(5). doi:10.1371/journal.pone.0177264

19. L M, M P, H K, et al. Arterial Stiffness Assessment by Shear Wave Elastography and Ultrafast Pulse Wave Imaging: Comparison with Reference Techniques in Normotensives and Hypertensives. Ultrasound Med Biol. 2019;45(3):758-772. doi:10.1016/J.ULTRASMEDBIO.2018.10.032

20. Urban MW, Pislaru C, Nenadic IZ, Kinnick RR, Greenleaf JF. Measurement of viscoelastic properties of in vivo swine myocardium using Lamb Wave Dispersion Ultrasound Vibrometry (LDUV). doi:10.1109/TMI.2012.2222656

21. Bouchard RR, Hsu SJ, Palmeri ML, Rouze NC, Nightingale KR, Trahey GE. Acoustic Radiation Force-Driven Assessment of Myocardial Elasticity Using the Displacement 
Ratio Rate (DRR) Method. Ultrasound Med Biol. 2011;37(7):1087-1100.

doi:10.1016/j.ultrasmedbio.2011.04.005

22. Bouchard RR, Hsu SJ, Wolf PD, Trahey GE. In vivo cardiac, acoustic-radiation-forcedriven, shear wave velocimetry. Ultrason Imaging. 2009;31(3):201-213. doi:10.1177/016173460903100305

23. Petrescu A, Santos P, Orlowska M, et al. Velocities of Naturally Occurring Myocardial Shear Waves Increase With Age and in Cardiac Amyloidosis. JACC CardiovasC Imaging. 2019. doi:10.1016/j.jcmg.2018.11.029

24. A P, S B, M C, et al. Shear Wave Elastography Using High-Frame-Rate Imaging in the Follow-Up of Heart Transplantation Recipients. JACC Cardiovasc Imaging. 2020;13(11):2304-2313. doi:10.1016/J.JCMG.2020.06.043

25. Nagueh SF, Smiseth OA, Appleton CP, et al. Recommendations for the Evaluation of Left Ventricular Diastolic Function by Echocardiography: An Update from the American Society of Echocardiography and the European Association of Cardiovascular Imaging. J Am Soc Echocardiogr. 2016;29:277-314. doi:10.1016/j.echo.2016.01.011

26. Lang RM, Badano LP, Mor-Avi V, et al. Recommendations for Cardiac Chamber Quantification by Echocardiography in Adults: An Update from the American Society of Echocardiography and the European Association of Cardiovascular Imaging. J Am Soc Echocardiogr. 2015;28(1):1-39.e14. doi:10.1016/J.ECHO.2014.10.003

27. Vos HJ, van Dalen BM, Bosch JG, van der Steen AFW, de Jong N. Myocardial passive shear wave detection. In: 2015 IEEE International Ultrasonics Symposium (IUS). IEEE; 2015:1-4. doi:10.1109/ULTSYM.2015.0152

28. Vejdani-Jahromi M, Freedman J, Nagle M, Kim YJ, Trahey GE, Wolf PD. Quantifying Myocardial Contractility Changes Using Ultrasound-Based Shear Wave Elastography. J Am Soc Echocardiogr. 2017;30(1):90-96. doi:10.1016/j.echo.2016.10.004

29. Oommen B, Karamanoglu M, Kovács SJ. Modeling time varying elastance: The meaning of "load-independence." Cardiovasc Eng. 2003;3(4):123-130. doi:10.1023/B:CARE.0000018825.33151.bc

30. Pernot M, Lee WN, Bel A, et al. Shear Wave Imaging of Passive Diastolic Myocardial Stiffness: Stunned Versus Infarcted Myocardium. JACC Cardiovasc Imaging. 2016.

31. Lee WN, Pernot M, Couade M, et al. Mapping myocardial fiber orientation using echocardiography-based shear wave imaging. IEEE Trans Med Imaging. 2012;31(3):554-562. doi:10.1109/TMI.2011.2172690 
32. Song P, Bi X, Mellema DC, et al. Pediatric Cardiac Shear Wave Elastography for Quantitative Assessment of Myocardial Stiffness: A Pilot Study in Healthy Controls. Ultrasound Med Biol. 2016;42(8):1719-1729. doi:10.1016/j.ultrasmedbio.2016.03.009

33. Pernot M, Couade M, Mateo P, Crozatier B, Fischmeister R, Tanter M. Real-Time Assessment of Myocardial Contractility Using Shear Wave Imaging. 2011. doi:10.1016/j.jacc.2011.02.042

34. Kanai $\mathrm{H}$. Propagation of spontaneously actuated pulsive vibration in human heart wall and in vivo viscoelasticity estimation. IEEE Trans Ultrason Ferroelectr Freq Control. 2005;52(11):1931-1942. doi:10.1109/TUFFC.2005.1561662

35. Cvijic M, Bézy S, Petrescu A, et al. Interplay of Cardiac Remodelling and Myocardial Stiffness in Hypertensive Heart Disease.

36. Salles S, Aase SA, Bjastad T, Lovstakken L, Torp H. Clutter filter wave imaging (CFWI): A new way to visualize and detect mechanical waves propagation. In: 2017 IEEE International UItrasonics Symposium (IUS). IEEE; 2017:1-4. doi:10.1109/ULTSYM.2017.8092459

37. Santos P, Haugen G, Lovstakken L, Samset E, D'hooge J. High frame rate 3D tissue velocity imaging using sub-aperture beamforming: A pilot study in vivo. In: 2016 IEEE International Ultrasonics Symposium (IUS). IEEE; 2016:1-4.

doi:10.1109/ULTSYM.2016.7728385

38. Provost J, Papadacci C, Arango JE, et al. 3D ultrafast ultrasound imaging in vivo. Phys Med Biol. 2014;59(19):L1-L13. doi:10.1088/0031-9155/59/19/L1

39. Salles S, Espeland T, Molares A, et al. 3D Myocardial Mechanical Wave Measurements: Toward In Vivo 3D Myocardial Elasticity Mapping. JACC CardiovasC Imaging. 2020. doi:10.1016/j.jcmg.2020.05.037

40. Papadacci C, Finel V, Villemain O, Tanter M, Pernot M. 4D ultrafast ultrasound imaging of naturally occurring shear waves in the human heart. IEEE Trans Med Imaging. 2020:1-1. doi:10.1109/TMI.2020.3020147

41. Voigt JU, Cvijic M. 2- and 3-Dimensional Myocardial Strain in Cardiac Health and Disease. JACC Cardiovasc Imaging. 2019;12(9):1849-1863. doi:10.1016/j.jcmg.2019.01.044

42. P J, J P, H L, et al. High-Frame-Rate Speckle-Tracking Echocardiography. IEEE Trans Ultrason Ferroelectr Freq Control. 2018;65(5):720-728.

doi:10.1109/TUFFC.2018.2809553 
43. MV A, C M, K A, et al. High-Frame-Rate Deformation Imaging in Two Dimensions Using Continuous Speckle-Feature Tracking. Ultrasound Med Biol. 2016;42(11):26062615. doi:10.1016/J.ULTRASMEDBIO.2016.07.009

44. Orlowska M, Ramalli A, Bezy S, Meacci V, Voigt J-U, D’hooge J. In-Vivo Comparison of Multiline Transmission and Diverging Wave Imaging for High Frame Rate Speckle Tracking Echocardiography. IEEE Trans Ultrason Ferroelectr Freq Control. 2020.

45. Orlowska M, Ramalli A, Petrescu A, et al. A Novel 2-D Speckle Tracking Method for High-Frame-Rate Echocardiography. IEEE Trans Ultrason Ferroelectr Freq Control. 2020;67(9):1764-1775. doi:10.1109/TUFFC.2020.2985451

46. Lu MT, Ferencik M, Roberts RS, et al. Noninvasive FFR Derived From Coronary CT Angiography: Management and Outcomes in the PROMISE Trial. JACC CardiovasC Imaging. 2017;10(11):1350-1358. doi:10.1016/j.jcmg.2016.11.024

47. Maresca D, Correia M, Villemain O, et al. Noninvasive Imaging of the Coronary Vasculature Using Ultrafast Ultrasound. JACC Cardiovasc Imaging. August 2017. doi:10.1016/j.jcmg.2017.05.021

48. Demene C, Deffieux T, Pernot M, et al. Spatiotemporal Clutter Filtering of Ultrafast Ultrasound Data Highly Increases Doppler and fUltrasound Sensitivity. IEEE Trans Med Imaging. 2015;34(11):2271-2285. doi:10.1109/TMI.2015.2428634

49. M C, D M, G G, et al. Quantitative imaging of coronary flows using 3D ultrafast Doppler coronary angiography. Phys Med Biol. 2020;65(10):105013. doi:10.1088/1361-6560/ab8d78

50. Chapman J V. The Technical Aspects of Doppler Ultrasound. 1990:1-34. doi:10.1007/978-94-009-0647-1_1

51. Pedrizzetti G, Martiniello AR, Bianchi V, D’Onofrio A, Caso P, Tonti G. Cardiac fluid dynamics anticipates heart adaptation. J Biomech. 2015;48(2):388-391. doi:10.1016/j.jbiomech.2014.11.049

52. Sengupta PP, Pedrizzetti G, Kilner PJ, et al. Emerging Trends in CV Flow Visualization. JACC Cardiovasc Imaging. 2012;5(3):305-316. doi:10.1016/j.jcmg.2012.01.003

53. Prinz C, Faludi R, Walker A, et al. Can echocardiographic particle image velocimetry correctly detect motion patterns as they occur in blood inside heart chambers? A validation study using moving phantoms. Cardiovasc Ultrasound. 2012;10(1):24. doi:10.1186/1476-7120-10-24 
54. Faludi R, Szulik M, D'hooge J, et al. Left ventricular flow patterns in healthy subjects and patients with prosthetic mitral valves: An in vivo study using echocardiographic particle image velocimetry. J Thorac Cardiovasc Surg. 2010;139(6):1501-1510. doi:10.1016/J.JTCVS.2009.07.060

55. Gao H, Claus P, Amzulescu M-S, Stankovic I, D'hooge J, Voigt J-U. How to optimize intracardiac blood flow tracking by echocardiographic particle image velocimetry? Exploring the influence of data acquisition using computer-generated data sets. Eur Heart J Cardiovasc Imaging. 2012;13(6):490-499. doi:10.1093/ejechocard/jer285

56. Leow CH, Bazigou E, Eckersley RJ, Yu ACH, Weinberg PD, Tang M-X. Flow Velocity Mapping Using Contrast Enhanced High-Frame-Rate Plane Wave Ultrasound and Image Tracking: Methods and Initial inÂ Vitro and inÂ Vivo Evaluation. Ultrasound Med Biol. 2015;41(11):2913-2925. doi:10.1016/J.ULTRASMEDBIO.2015.06.012

57. Fadnes S, Wigen MS, Nyrnes SA, Lovstakken L. In Vivo Intracardiac Vector Flow Imaging Using Phased Array Transducers for Pediatric Cardiology. IEEE Trans Ultrason Ferroelectr Freq Control. 2017;64(9):1318-1326.

doi:10.1109/TUFFC.2017.2689799

58. Fadnes S, Nyrnes SA, Torp H, Lovstakken L. Shunt flow evaluation in congenital heart disease based on two-dimensional speckle tracking. Ultrasound Med Biol. 2014;40(10):2379-2391. doi:10.1016/j.ultrasmedbio.2014.03.029

59. Nyrnes SA, Fadnes S, Wigen MS, Mertens L, Lovstakken L. Blood Speckle-Tracking Based on High-Frame Rate Ultrasound Imaging in Pediatric Cardiology. J Am Soc Echocardiogr. 2020;33(4):493-503.e5. doi:10.1016/J.ECHO.2019.11.003

60. Wigen MS, Fadnes S, Rodriguez-Molares A, et al. 4-D Intracardiac Ultrasound Vector Flow Imaging-Feasibility and Comparison to Phase-Contrast MRI. IEEE Trans Med Imaging. 2018;37(12):2619-2629. doi:10.1109/TMI.2018.2844552

61. Wigen $M$, Lovstakken L. In vivo three-dimensional intra-cardiac vector flow imaging using a 2D matrix array transducer. In: 2016 IEEE International Ultrasonics Symposium (IUS). IEEE; 2016:1-4. doi:10.1109/ULTSYM.2016.7728690

62. SA N, S F, MS W, L M, L L. Blood Speckle-Tracking Based on High-Frame Rate Ultrasound Imaging in Pediatric Cardiology. J Am Soc Echocardiogr. 2020;33(4):493503.e5. doi:10.1016/J.ECHO.2019.11.003 



\section{Figure Legends}

Figure [F1]: Shear Wave Imaging with intrinsic or external excitation. (A) External excitation by a strong focused ultrasound impulse (red) induces a translational wave (shear wave, green)) that propagates along the myocardium. The propagation of the wave is then vizualized by high frame rate imaging. The propagation velocity of a shear wave is directly related to the stiffness of the tissue. Physiologic events, such as aortic or mitral valve closure (red) can also generate a shear wave.

Figure [F2]: Assessment of a shear wave induced in the septum by aortic valve closure. (A) Parasternal long axis view. The colour encodes Doppler based tissue acceleration in the septum. The white dotted line represents the orientation of the anatomical M-mode. (B) Anatomic M-mode, derived from the white dotted line in (A). Colour coding as in (A). A pronounced shear wave (light blue/yellow) propagates from the anteroseptal base towards the apex immediately after aortic valve closure (AVC) with a velocity of $3.6 \mathrm{~m} / \mathrm{s}$ (From Santos et al. ${ }^{13}$, with permission).

Figure [F3]: Myocardial stiffness in healthy volunteers of different age and patients with HCM.

Comparison of myocardial stiffness in healthy volunteers (HV, green bar) and heart failure patients with hypertrophic cardiomyopathy ( $\mathrm{HCM}$, red bar). A strong increase in myocardial stifness was found with disease and increasing age. Values denote the myocardial stiffness in $\mathrm{kPa}$. (From Villemain et al. ${ }^{16}$, with permission)

\section{Figure [F4]: Shear wave propagation velocities in patients after HTX.}

CMR T1 mapping of the mid left ventricular segment of the anteroseptal wall and acceleration M-mode map of the anteroseptal wall in three heart transplant recipients (HTx13). Note that shear wave velocities at mitral valve closure (MVC) increase with increasing native $\mathrm{T} 1$ value. The highlighted region of the ECG indicates the time interval covered by the M-mode map. $\mathrm{HTx}=$ heart transplant recipient; MVC = mitral valve closure. (From Petrescu et al. ${ }^{24}$, with permission)

Figure [F5]: 3D visualization of mechanical wave propagation along the left ventricle at atrial systole. The normalized tissue acceleration is mapped in 3D over time. The white arrow indicates the instantaneous maximum of the acceleration. (from Salles et al. ${ }^{16}$, with permission) 
Figure [F6]: Ultrafast color Doppler imaging of intramural coronary vasculature. Flow in epicardial and intramyocardial vessels an open-chest swine experiment. Vessels below 100 $\mu \mathrm{m}$ remain below resolution. The scale bar represents $3 \mathrm{~mm}$. A and $B$ longitudinal images, $C$ and $D$ short axis images. Panels $A$ and $C$ : in a systole, venous blood flow moves upwards from the endocardium to the epicardium (red) and is collected in the epicardial veins. Panels $\mathrm{B}$ and $\mathrm{D}$ : in diastole, arterial blood from the epicardial vessels flows downwards (blue) in the myocardium. (from Maresca et al. ${ }^{47}$, with permission)

Figure [F7]: Advanced cardiac flow imaging. The figure shows a 3D representation of the flow path lines in three parts of the cardiac cycle corresponding to diastole, diastasis and systole, respectively, by using blood speckle tracking. Complex swirling flow patterns can be displayed throughout the cardiac cycle. (from Wigen et al. ${ }^{60}$, with permission) 


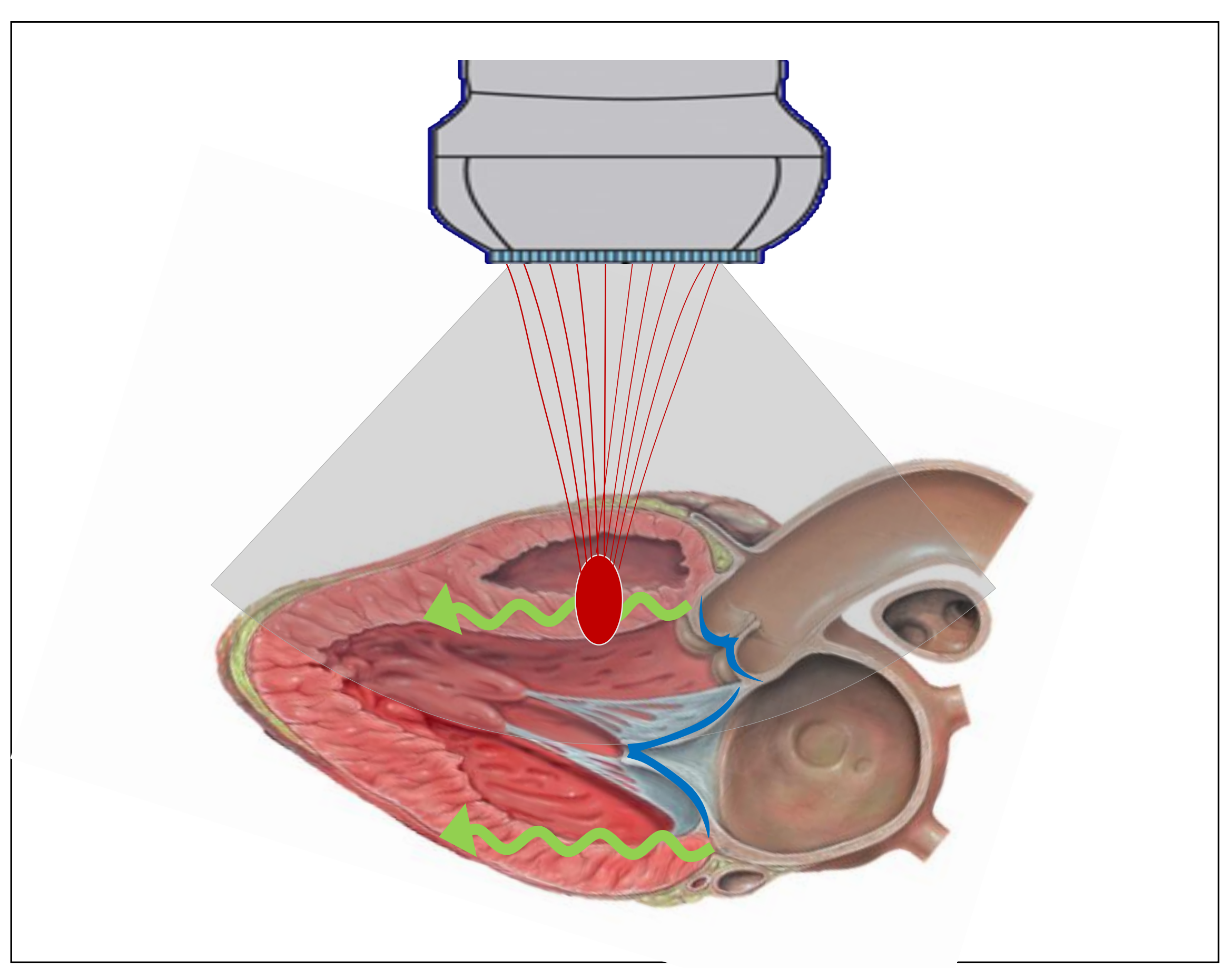


A.
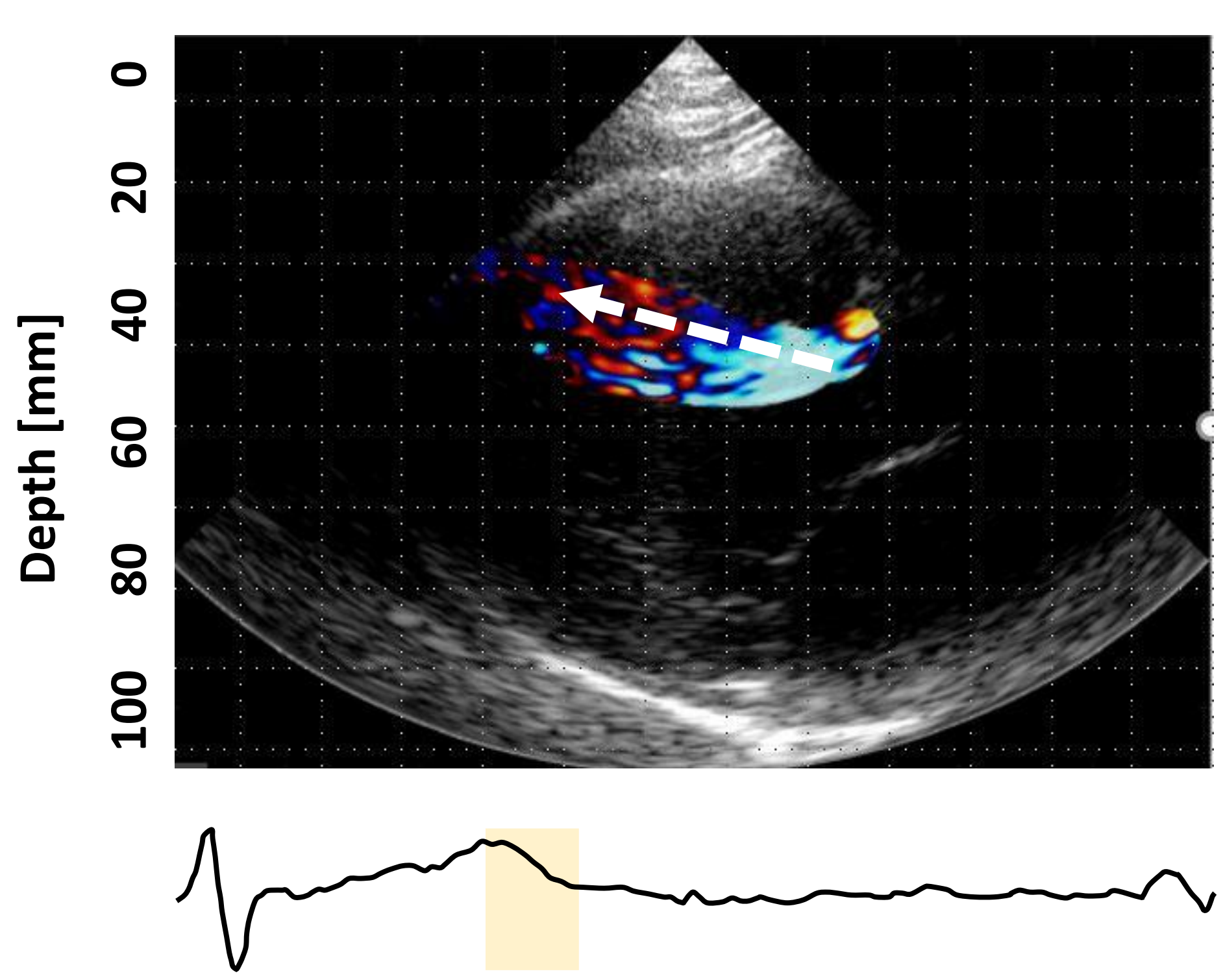

B.

$S W$ velocity $=3.6 \mathrm{~m} / \mathrm{s}$

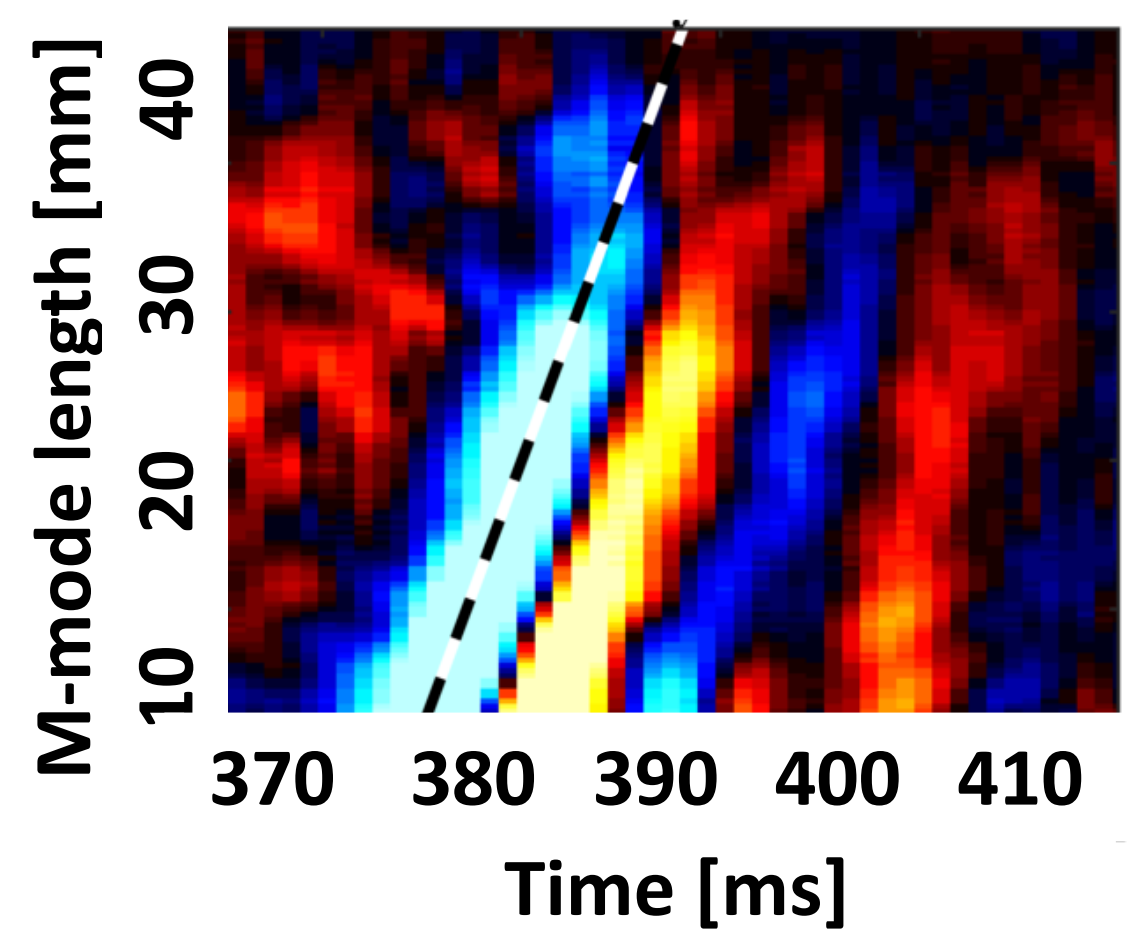

$\begin{array}{lllllll}-3 & -2 & -1 & 0 & 1 & 2 & 3\end{array}$ Acceleration $\left[\mathrm{m} / \mathrm{s}^{2}\right]$ 


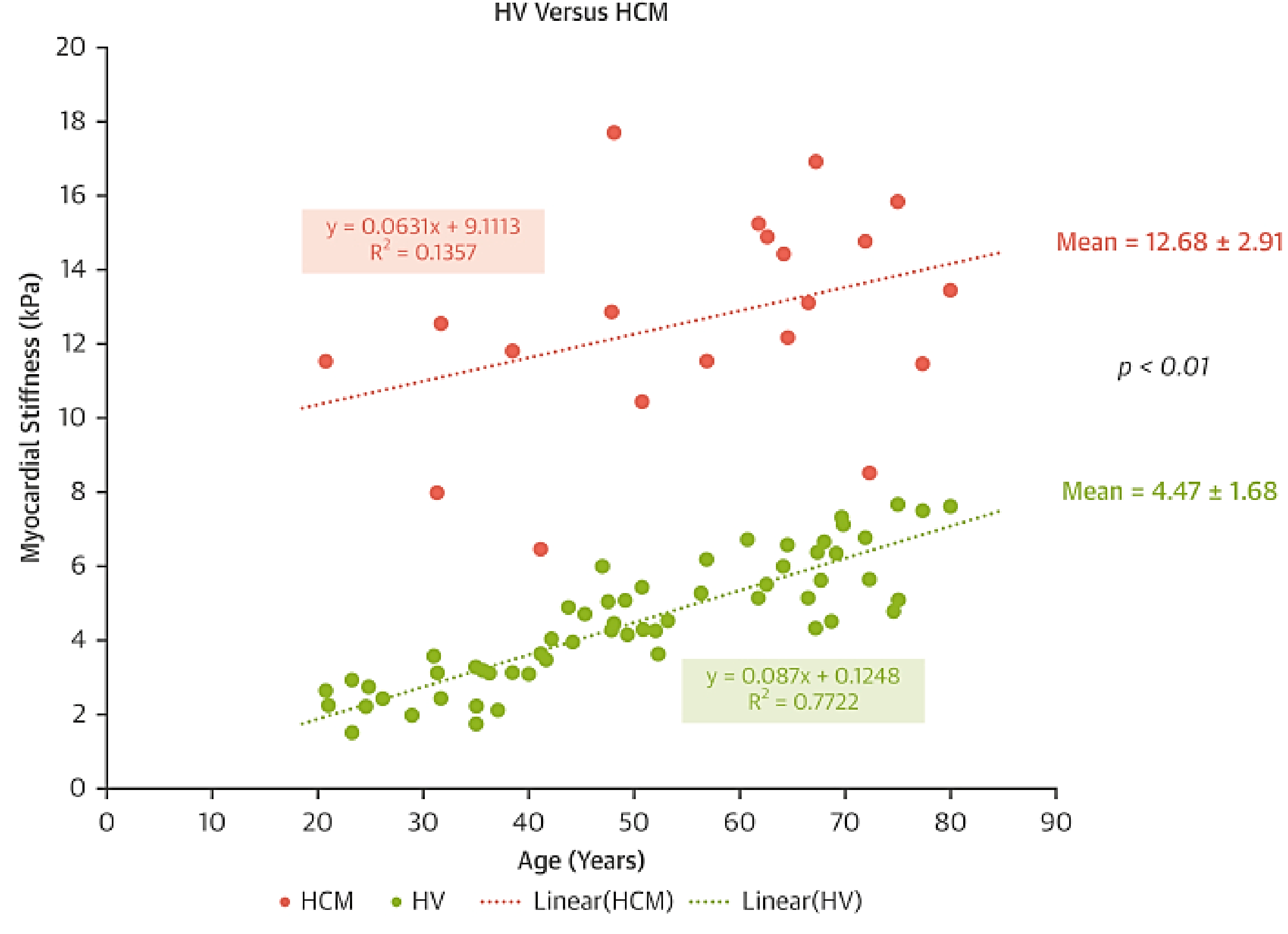


HTx 1

HTx 2

HTx 3

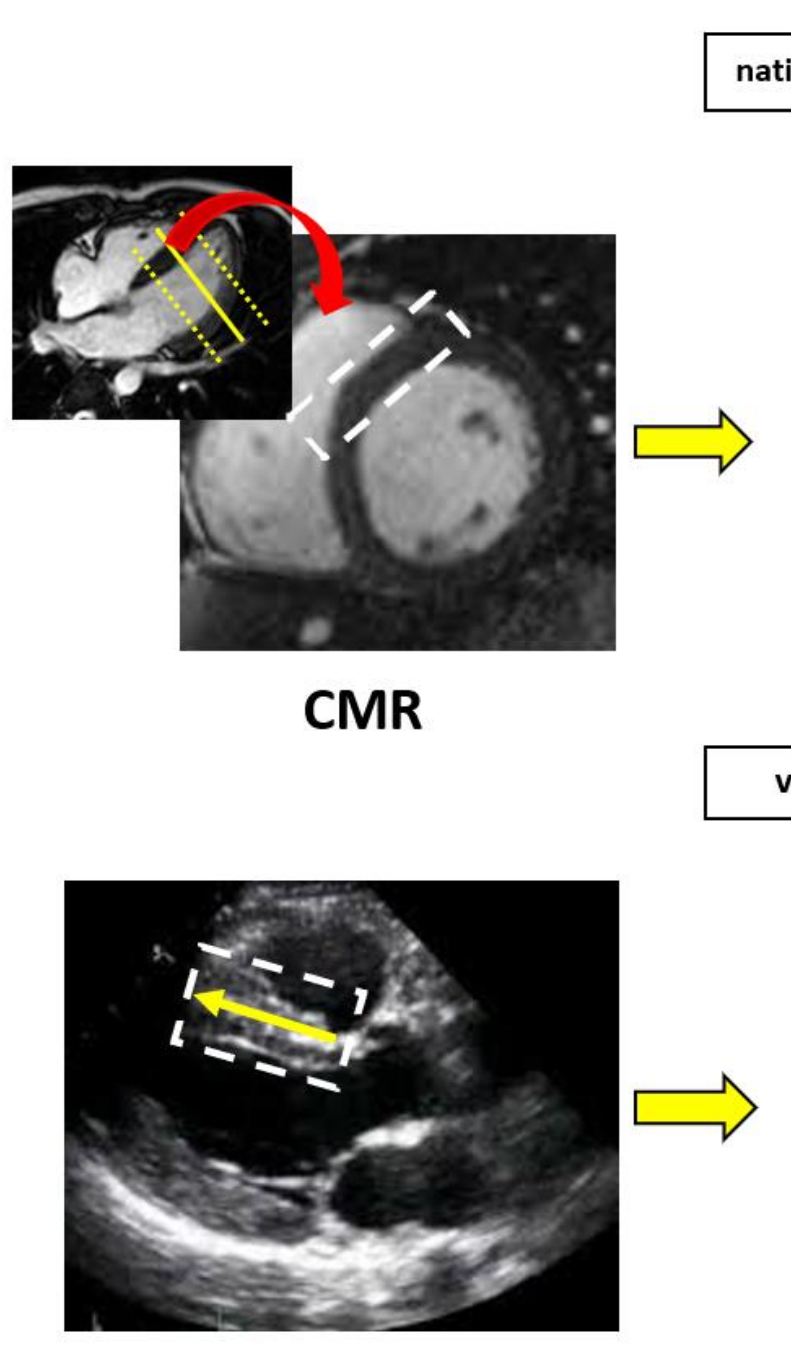

SWI

ECG

\section{nativeT1 $968 \mathrm{~ms}$}

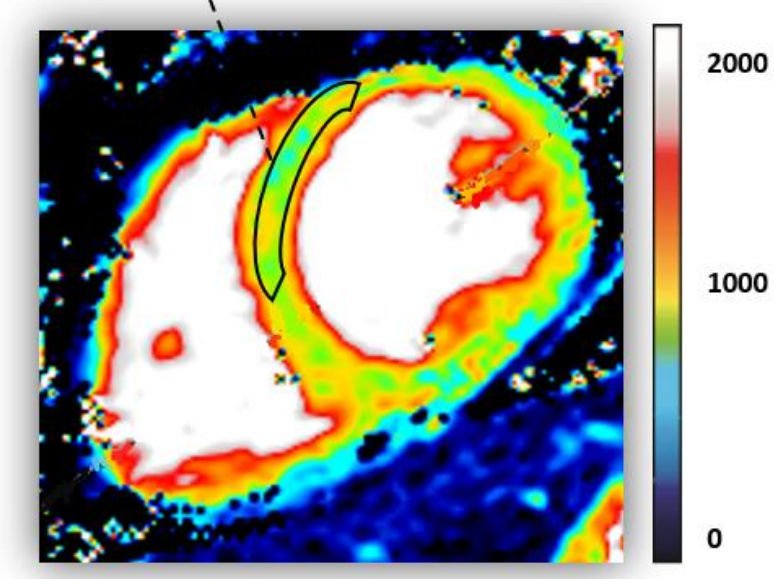

$$
v=3.2 \mathrm{~m} / \mathrm{s}
$$
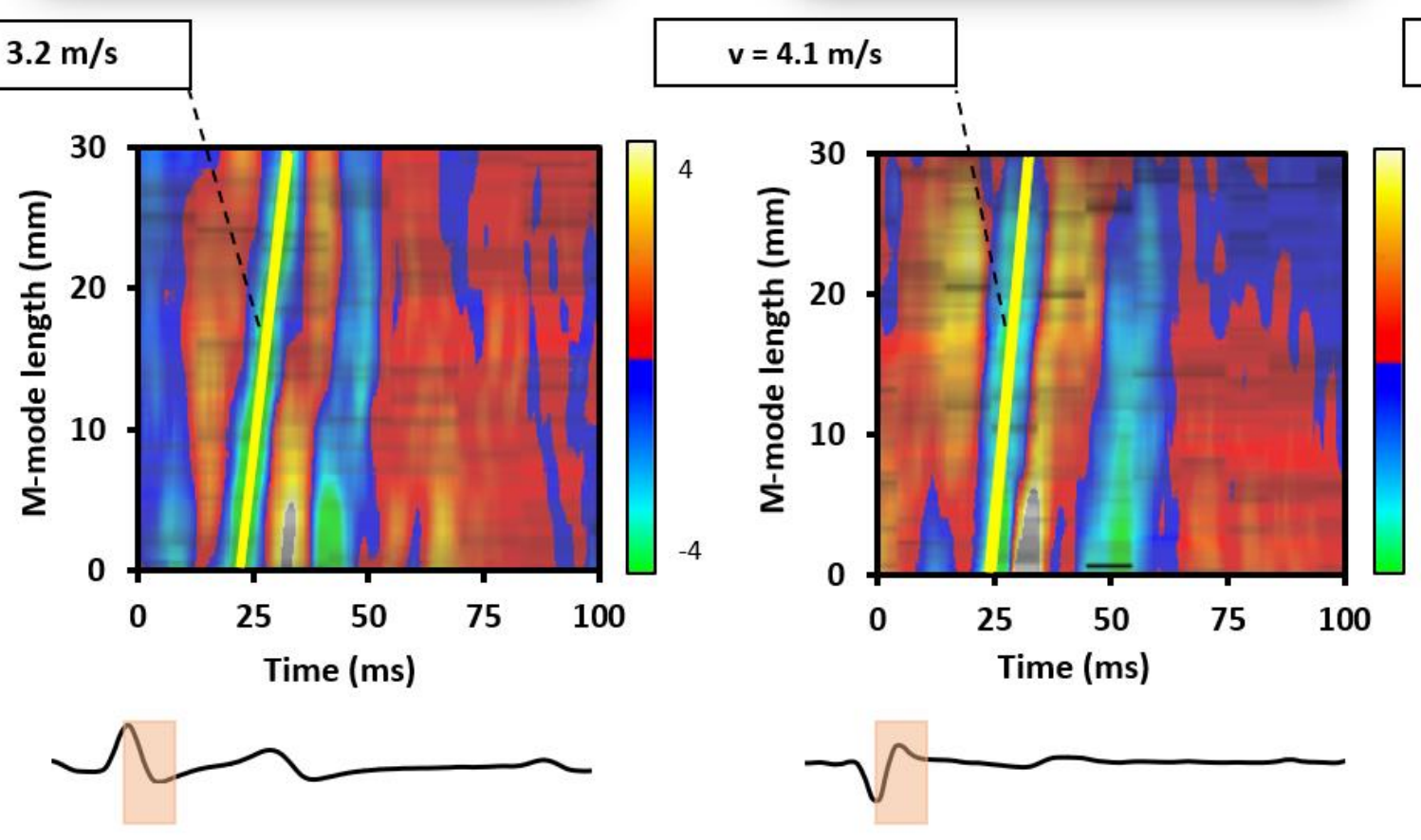

nativeT1 $1020 \mathrm{~ms}$
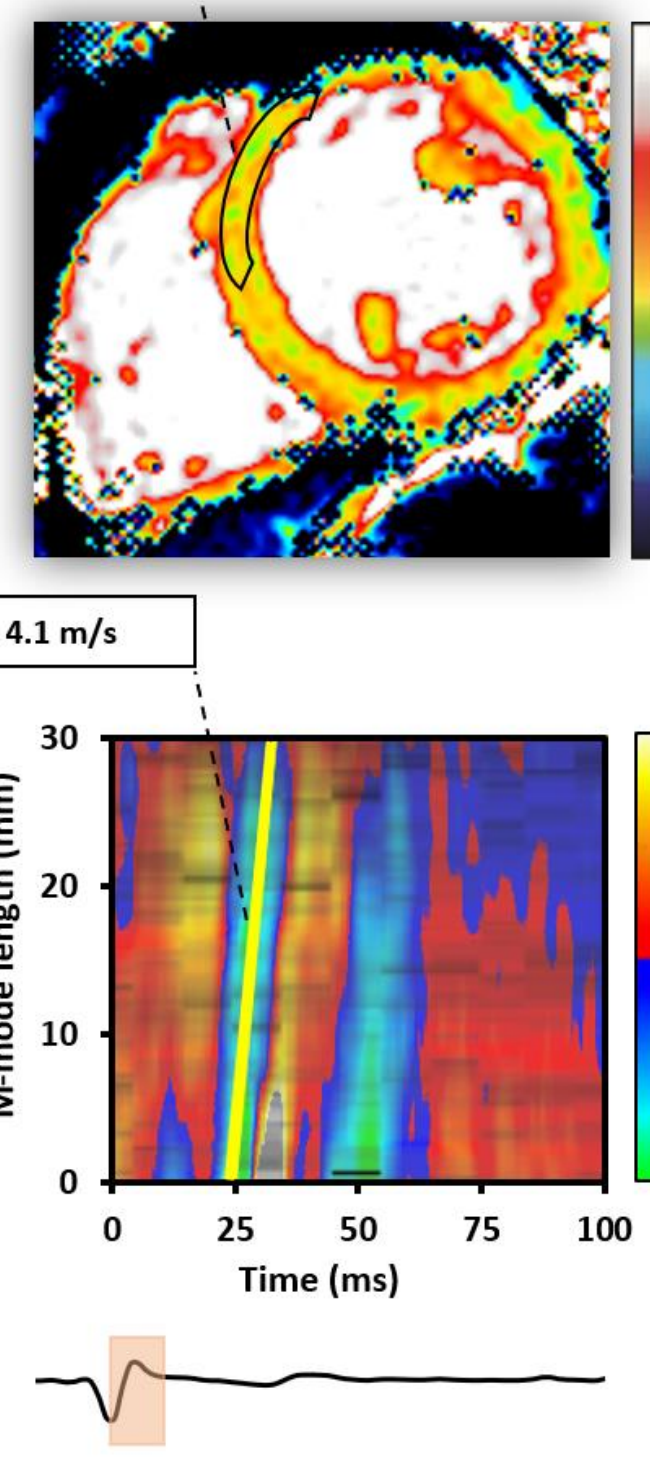

nativeT1 $1060 \mathrm{~ms}$

2000

1000

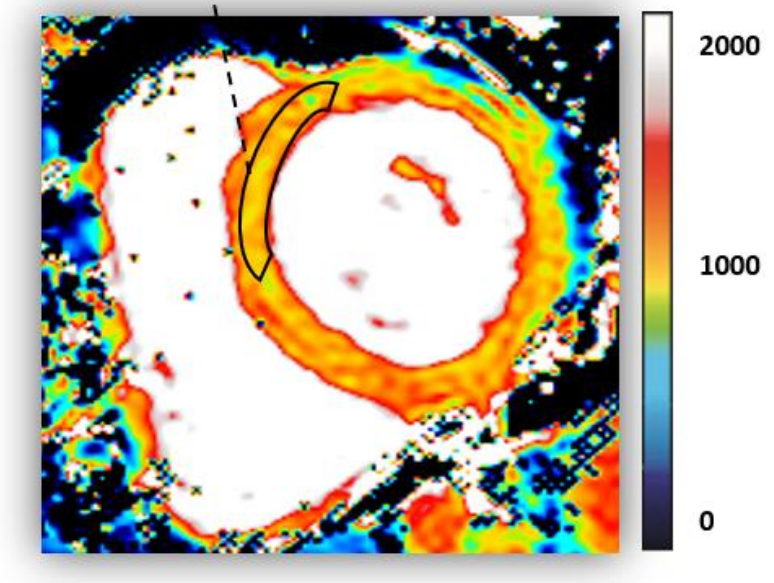

$=7.7 \mathrm{~m} / \mathrm{s}$
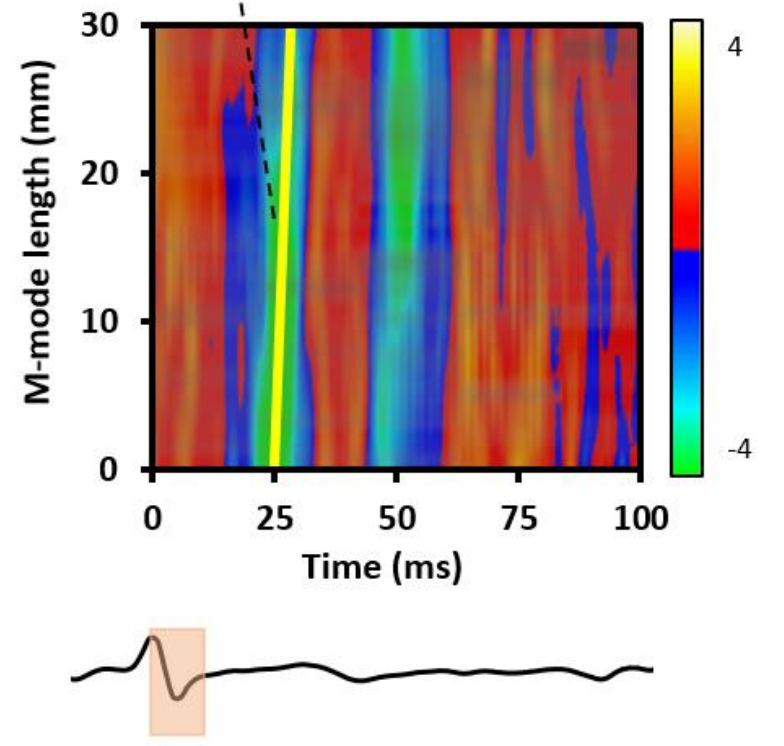


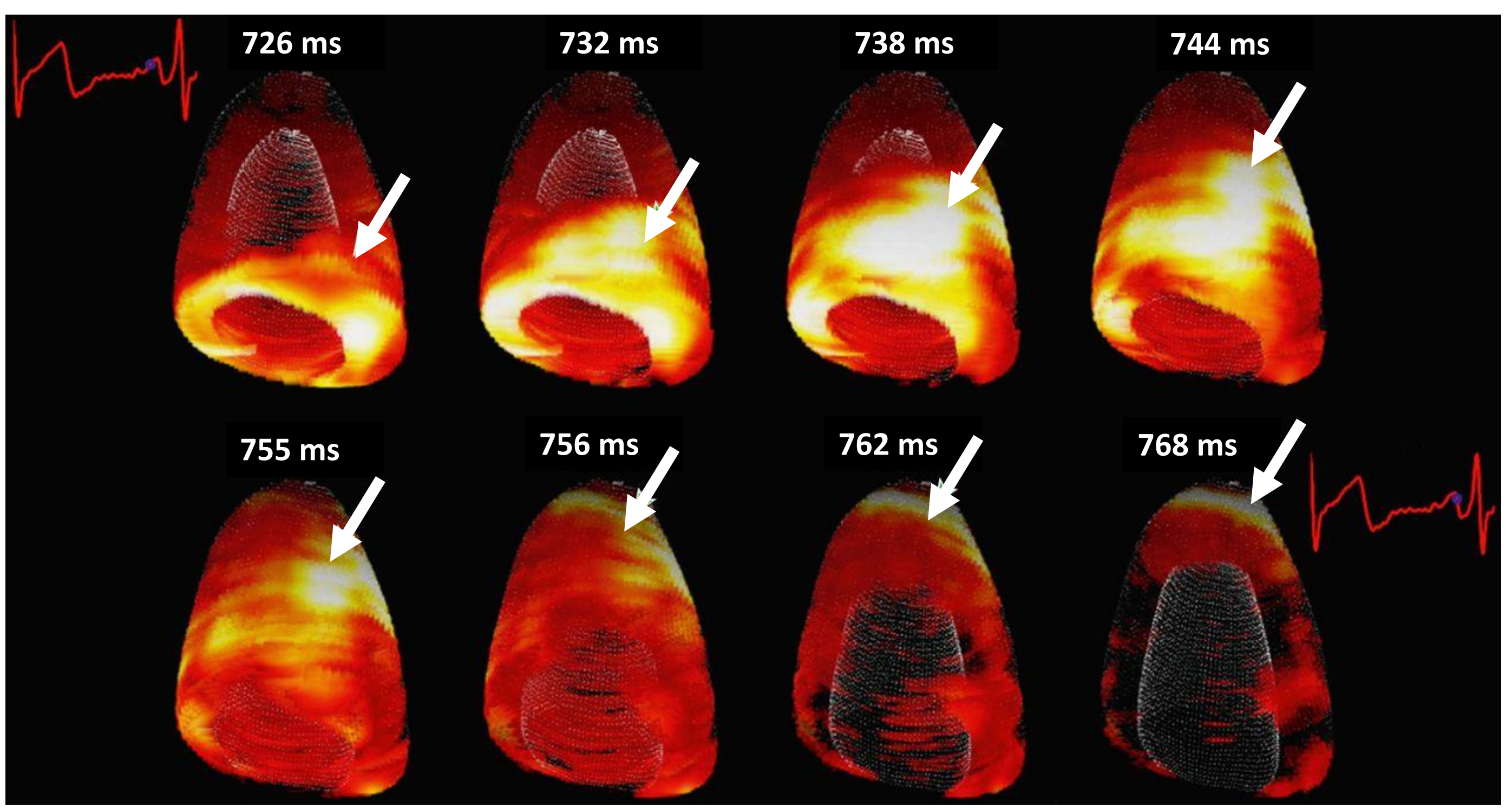




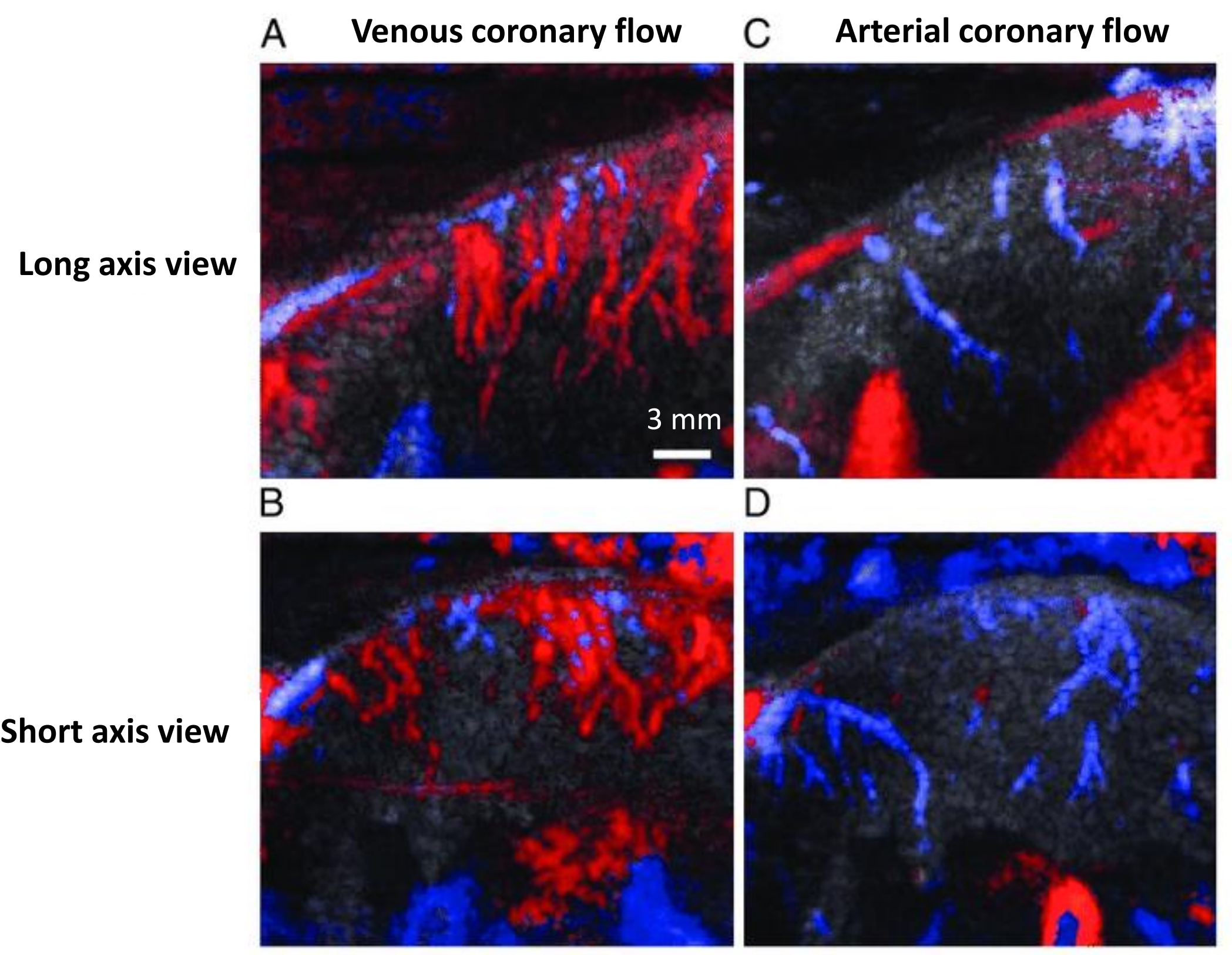




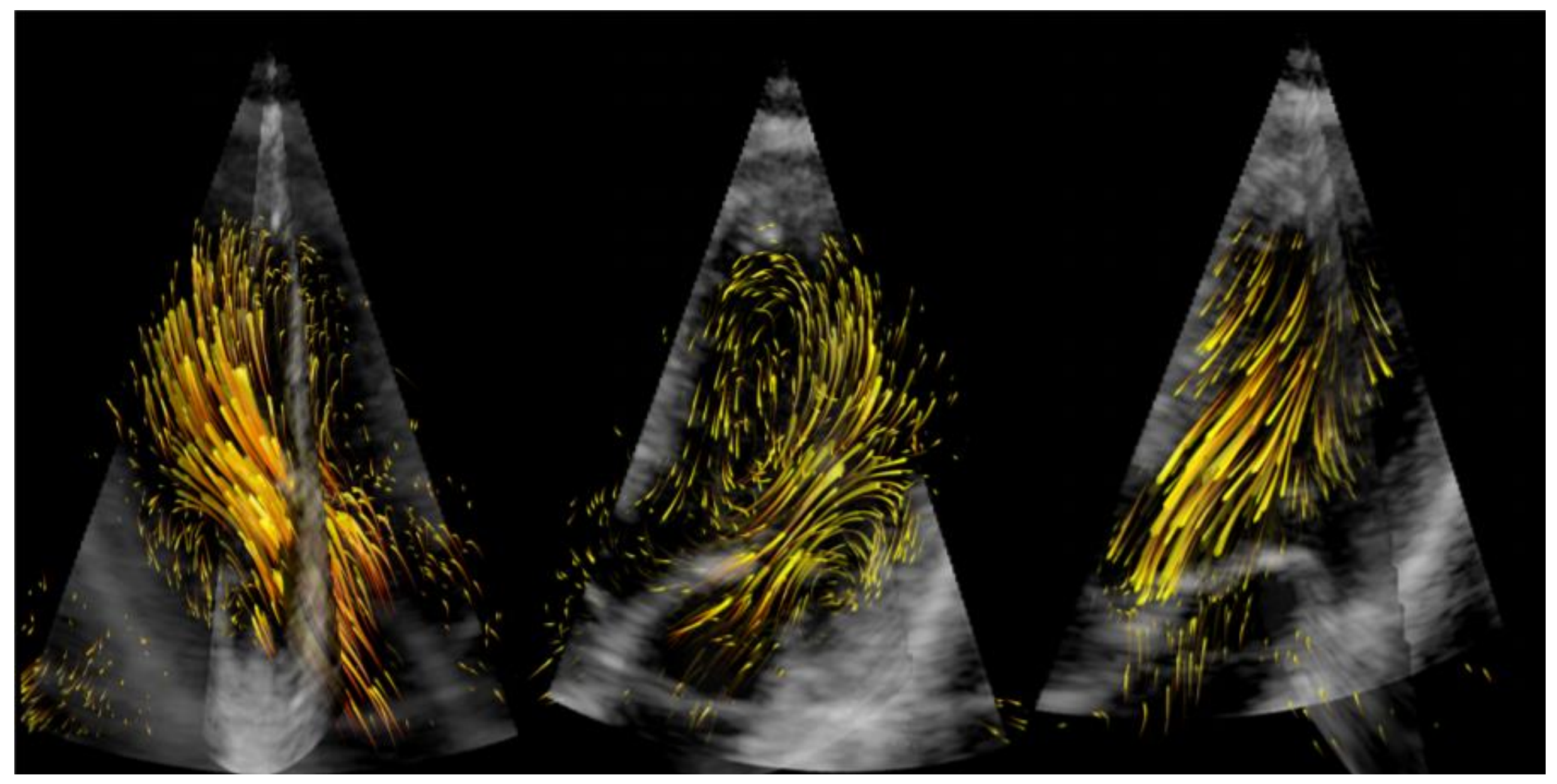

